\title{
El efecto visual y la necesidad de la determinación de un método de blanqueamiento superficial de la nuez de macadamia
}

\section{(The visual effect and the necessity of determinating the superficial whitening method of the macadamia's nut)}

\author{
María José Jijón ${ }^{1}$ Teresa Guerrero Villegas ${ }^{1}$, Adriana Barahona ${ }^{1}$ \\ Pablo Aguilar Barriga ${ }^{1}$
}

\begin{abstract}
Resumen:
La nuez de macadamia es considerada "la reina de las nueces", por su alto contenido energético y valor nutritivo. Cierto tipo de nueces se considera poco comercial debido a su color, por lo cual el objetivo del presente estudio fue determinar un método de blanqueo superficial utilizando aditivos que no afecten el sabor, olor y textura de la nuez. Se emplearon tres aditivos alimentarios como agentes blanqueadores: ácido cítrico al $1 \%$ durante 2 minutos y al $3 \%$ durante 1 minuto, ácido ascórbico al $0.1 \%$ durante 1 minuto y al $0.3 \%$ durante 3 minutos, y bicarbonato de sodio al 5 y $10 \%$ durante 1 minuto. Para todos los tratamientos se realizaron análisis de color, humedad de la nuez, análisis sensorial de olor, sabor y textura. Las nueces se blanquearon superficialmente después de secarse. Los resultados indican que el mejor tratamiento para blanquear la nuez de macadamia es el ácido ascórbico al $0.3 \%$; con ello se consigue aportar a la nuez un color más homogéneo y natural, libre de manchas negruzcas.
\end{abstract}

Palabras clave: Antioxidante; aditivo; cualidad sensorial; agente blanqueador.

\begin{abstract}
:
Macadamia nut is considered "the queen of nuts", because of its high energy content and nutritional value. Certain types of nuts are considered to be non-commercial due to their color; therefore, the aim of this study was determining a method of surface bleaching using additives that do not affect the taste, smell and texture of the walnut. Color is a sensory quality of food; it's an indicator and a critical quality factor in fresh and processed products. Three food additives were used as bleaching agents: $1 \%$ citric acid for 2 minutes and $3 \%$ for 1 minute, $0.1 \%$ ascorbic acid for 1 minute and $0.3 \%$ for 3 minutes, and 5 and $10 \%$ sodium bicarbonate for 1 minute. For all treatments, color analysis, walnut moisture, sensory analysis of odor, taste and texture were carried out. Walnuts were bleached superficially after drying. The results indicate that the best treatment for bleaching macadamia nut is ascorbic acid at $0.3 \%$, with this it is possible to add a more homogeneous and natural color to the nut, free of blackish spots.
\end{abstract}

Keywords: Antioxidant; additive; sensory quality; bleaching agent.

\section{Introducción}

La macadamia (Macadamia integrifolia) es originaria de Australia. Fue introducida en Ecuador en 1976 y su fruto seco, la nuez, es considerado un cultivo exótico, por su alto contenido de ácidos grasos monoinsaturados (Rodríguez Millán, Silva Ramírez \& Carrillo Inungaray, 2011), además de su delicado sabor. Aulestia (2010), manifiesta que el cultivo de macadamia en Ecuador es relativamente nuevo; las plantaciones productoras se encuentran en zonas subtropicales de la Costa, como Quinindé, Santo

\footnotetext{
1 Universidad Tecnológica Equinoccial, Quito - Ecuador (\{adriana.barahona, pablo.aguilar, esthela.guerrero, jcmj41290\} @ute.edu.ec)
} 
Domingo de los Tsáchilas, Puerto Quito, Pedro Vicente Maldonado, Quevedo, La Maná, La Concordia, San Lorenzo, y en la región Amazónica ciudades como Francisco de Orellana (EI Coca), Tena y Lago Agrio.

La nuez de macadamia es de forma esférica, de color blanquecino o crema, reconocida como la nuez más fina del mundo debido al delicado sabor que posee, lo que la ubica en el mercado de los productos gourmet (Erazo, 2006). El fruto es una nuez comestible de color blanco cremoso, cuyo diámetro oscila entre 12 y $20 \mathrm{~mm}$; está encerrada en una fuerte cáscara o concha lisa de color café, que a su vez se encuentra dentro de una cáscara verde suave que se abre cuando el fruto está maduro. Las diferentes variedades de Macadamia integrifolia producen nueces cuyo color puede ir desde blanco crema a habano claro. Las nueces más oscuras no satisfacen visualmente a los clientes, lo que dificulta su venta y puede causar pérdidas económicas a sus productores (Lavin et al., 2001).

Para Reyes y Moreno (2009), las nueces de macadamia, al igual que otros frutos secos, están libres de colesterol y son una gran fuente de fibra; poseen un alto contenido en grasa, ya que casi el $80 \%$ es grasa monoinsaturada, un tipo de grasa buena que ayuda a reducir el colesterol total y el colesterol malo o LDL y aumenta el colesterol HDL o colesterol bueno. Es uno de los pocos alimentos que contienen Omega-7 o ácido palmitoleico, reduce el colesterol, y el riesgo de enfermedades del corazón, pero también puede afectar el metabolismo, frenando el apetito y ayudando a quemar grasa más rápido. La composición nutricional por cada $100 \mathrm{~g}$ de porción comestible es de: 718 $\mathrm{kcal}, 7.91 \mathrm{~g}$ de proteína, $13.82 \mathrm{~g}$ de carbohidratos, $1.05 \mathrm{~g}$ de almidón, $4.57 \mathrm{~g}$ de azúcares simples, $75.77 \mathrm{~g}$ de lípidos, $8.6 \mathrm{~g}$ de fibra total, $1.36 \mathrm{~g}$ de ceniza y $2.88 \mathrm{~g}$ de agua (Gil \& Ruíz, 2010).

Se conocen diferentes variedades de macadamia que presentan diversos colores, algunas de ellas no satisfacen visualmente a los clientes por lo que se dificulta su venta en el mercado, para reducirla completamente, la mejor opción consiste en escaldarlas o blanquearlas; el objetivo del presente estudio fue determinar el método óptimo de blanqueo superficial para la nuez de macadamia y establecer su porcentaje de humedad para exponerla a un proceso de secado, realizar la caracterización sensorial de sabor, olor, textura y someterla al proceso de blanqueamiento.

El color es una cualidad sensorial de los alimentos, es un indicador y un factor crítico de calidad en algunos productos frescos y procesados. Existen algunos factores que modifican o determinan el color de un alimento, como puede ser la deshidratación, evaporación, ahumado o el blanqueamiento. Para determinar un método de blanqueo superficial para la nuez de macadamia, se utilizaron tres aditivos alimentarios como agentes blanqueadores: ácido cítrico al $1 \%$ y al $3 \%$; ácido ascórbico al $0.1 \%$ y al $0.3 \%$ y bicarbonato de sodio al $5 \%$ y $10 \%$ los resultados de los análisis concluyen que el mejor tratamiento para blanquear la nuez de macadamia es el ácido ascórbico al 0,3\%.

\section{Metodología}

\subsection{Material Vegetal y procesamiento}

Según el Instituto Ecuatoriano de Normalización. (1995), en la norma NTE INEN 1 233:95, de muestreo para granos y cereales, en los ensayos se utilizó $6.3 \mathrm{~kg}$ de nuez macadamia; se dividió en $300 \mathrm{~g}$, para cada tratamiento de la siguiente manera: $100 \mathrm{~g}$ para realizar la caracterización física de la nuez de macadamia, $10 \mathrm{~g}$ para la determinación de la humedad después del proceso de secado y $190 \mathrm{~g}$ para realizar el análisis sensorial. Según FAO (2006), para reducir la humedad de la nuez de macadamia a valores entre 3 y $1.5 \%$, se realiza un proceso de secado que ayuda en el momento de romper la cáscara o concha lisa de color café. Para que la almendra no sufra daño y no se quede pegada a la concha, existen dos maneras de secar la nuez, la primera se 
realiza de forma natural en tendales y en la segunda se utiliza el secado por calor en hornos.

\subsection{Soluciones Blanqueadoras}

Las soluciones blanqueadoras utilizadas fueron: ácido cítrico al 1 y $3 \%$, ácido ascórbico al 0.1 y $0.3 \%$ y bicarbonato de sodio al 5 y $10 \%$ (George et al., 1999), y se secó por un tiempo de 4 horas a $60^{\circ} \mathrm{C}$, para luego medir el color utilizando el colorímetro Konica Minolta Chroma Mater CR-200.

En la Tabla 1 se indica el agente blanqueador, la concentración de la solución blanqueadora y el tiempo de inmersión que se utilizó en cada tratamiento, cabe indicar que en las pruebas que se realizaron en la Escuela Politécnica Nacional se aplicaron los mismos tiempos para las tres soluciones blanqueadoras, una vez analizados los resultados se determinaron los mejores tiempos a utilizarse en la investigación.

Tabla 1. Agentes blanqueadores, concentración y tiempo de inmersión

\begin{tabular}{|c|c|c|c|}
\hline \multirow{2}{*}{ Tratamientos } & \multicolumn{3}{|c|}{ Muestras } \\
\cline { 2 - 4 } & $\begin{array}{c}\text { Agente } \\
\text { blanqueador }\end{array}$ & Concentración & $\begin{array}{c}\text { Tiempo } \\
\text { (min) }\end{array}$ \\
\hline T 1 & Ácido cítrico & $1 \%$ & 2 \\
\hline T 2 & Ácido cítrico & $3 \%$ & 1 \\
\hline T 3 & Ácido ascórbico & $0.1 \%$ & 1 \\
\hline T 4 & Ácido ascórbico & $0.3 \%$ & 3 \\
\hline T 5 & $\begin{array}{c}\text { Bicarbonato de } \\
\text { sodio }\end{array}$ & $5 \%$ & 1 \\
\hline T 6 & $\begin{array}{c}\text { Bicarbonato de } \\
\text { sodio }\end{array}$ & $10 \%$ & 1 \\
\hline T 7 & \multicolumn{3}{|c}{ Testigo } \\
\hline
\end{tabular}

\subsection{Caracterización Física y Análisis Sensorial}

La caracterización física fue realizada en el Departamento de Ciencias de Alimentos y Biotecnología de la Escuela Politécnica Nacional donde se midió el nivel de color obtenido utilizando el colorímetro Konica Minolta Chroma Mater CR-200. Este equipo se utilizó para obtener el valor de los parámetros de color (Carvajal, Aristizábal, Oliveros, \& Mejía, 2011).

La escala Cielab está basada en la respuesta de los observadores patrones a un estímulo luminoso, lo que quiere decir que se trata de imitar la respuesta humana promedio a las longitudes de onda de la luz y cómo una persona normal ve el color a través del espectro de luz visible. En este modelo, el espacio de color es un sistema coordenado cartesiano definido por tres coordenadas rectangulares $\left(L^{*}, a^{*}, b^{*}\right)$ de magnitudes dimensionales. La coordenada acromática $L^{*}$ es la luminosidad o claridad y representa si un color es oscuro, gris o claro, variando desde cero para un negro y hasta cien para un blanco. Las coordenadas cromáticas $a^{*} y b^{*}$ forman un plano perpendicular a $L^{*}$. La coordenada $a^{*}$ corresponde a rojo si $a^{*}$ es mayor a cero, 0 a verde se $a^{*}$ es menor a cero. La coordenada $b^{*}$ corresponde a amarillo si $b^{*}$ es mayor a cero, y a azul si $b^{*}$ es menor a cero (Carvajal, Aristizábal, Oliveros, \& Mejía, 2011).

Para el análisis sensorial se conformó un panel de 9 degustadores; los panelistas evaluaron olor, sabor y textura de las nueces en una escala de 1 a $7 ; 1$ corresponde a "me disgusta extremadamente" y 7, a "me gusta extremadamente". Los puntajes obtenidos en el análisis sensorial se calcularon mediante la prueba de Friedman (Barragán, 2012). 
Para el estudio se utilizó un Diseño Experimental completamente al azar (DCA), en este tipo de diseño todas las repeticiones experimentales se realizan en orden aleatorio completo, pues no se han tenido en cuenta otros factores de interés, de forma que los posibles efectos se repartan equitativamente entre los tratamientos, la investigación se realizó con siete tratamientos y tres repeticiones cada uno; el orden de los tratamientos fue aleatorio.

\subsection{Análisis Estadístico}

Los datos fueron analizados utilizando el paquete estadístico del programa Sedex (Software Estadístico Didáctico de Diseño Experimental) Versión 1.0. Se detectaron diferencias significativas en el análisis de resultados y se aplicó la prueba de Tukey con una significancia del $0.05 \%$.

\section{Resultados}

\subsection{Análisis del color de la nuez de macadamia}

Los instrumentos de medición de color, incluyendo espectrofotómetros y colorímetros, pueden cuantificar estos atributos de color fácilmente. Ellos determinan el color de un objeto dentro del espacio de color y muestran los valores para cada coordenada $L^{*}, a^{*}, y b^{*}$.

En la Tabla 2 se presentan los resultados obtenidos del análisis de color y humedad en la nuez de macadamia.

Tabla 2. Prueba de color y humedad de la nuez de macadamia después del proceso de secado

\begin{tabular}{|c|c|c|}
\hline \multirow{2}{*}{ Tratamientos } & Análisis & Análisis \\
\cline { 2 - 3 } & Luminosidad (L*) & Humedad (\%) \\
\hline Ácido cítrico 1\% & $76.22 \pm 1.50 \mathrm{ab}$ & $3.74 \pm 0.18^{\mathrm{b}}$ \\
\hline Ácido cítrico 3\% & $76.90 \pm 0.99 \mathrm{ab}$ & $3.19 \pm 0.37 \mathrm{ab}$ \\
\hline Ácido ascórbico 0,1\% & $77.54 \pm 2.71 \mathrm{ab}$ & $3.32 \pm 0.31 \mathrm{ab}$ \\
\hline Ácido ascórbico 0,3\% & $78.10 \pm 1.24^{\mathrm{a}}$ & $2.63 \pm 0.16 \mathrm{a}$ \\
\hline Bicarbonato de sodio 5\% & $73.53 \pm 1.86 \mathrm{~b}$ & $2.79 \pm 0.05 \mathrm{ab}$ \\
\hline Bicarbonato de sodio 10\% & $73.94 \pm 0.77^{\mathrm{ab}}$ & $2.94 \pm 0.68 \mathrm{ab}$ \\
\hline Testigo & $76.15 \pm 0.10^{\mathrm{ab}}$ & $2.78 \pm 0.38 \mathrm{ab}$ \\
\hline
\end{tabular}

Nota. a $\mathrm{L}^{*}$. La coordenada $\mathrm{a}^{*}$ corresponde a rojo si $\mathrm{a}^{*}$ es mayor a cero, o a verde si $\mathrm{a}^{*}$ es menor a cero. La coordenada $\mathrm{b}^{\star}$ corresponde a amarillo si b* es mayor a cero, y a azul si b* es menor a cero (Carvajal, Aristizábal, Oliveros, \& Mejía, 2011). Media \pm desviación estándar $(n=3)$.

\subsection{Análisis sensorial de olor}

En el análisis sensorial de la nuez de macadamia se evaluaron tres aspectos fundamentales: olor, sabor y textura. La Tabla 3 presenta los resultados obtenidos en el análisis sensorial de olor.

Para la textura, la prueba de Friedman no detecta diferencia significativa en los tratamientos tanto de ácido cítrico, ácido ascórbico y bicarbonato de sodio; el efecto de los agentes blanqueadores es el mismo. El ácido ascórbico y sus sales de sodio, potasio y calcio suelen usarse como aditivos antioxidantes de los alimentos. 
Tabla 3. Análisis sensorial de olor, sabor y textura

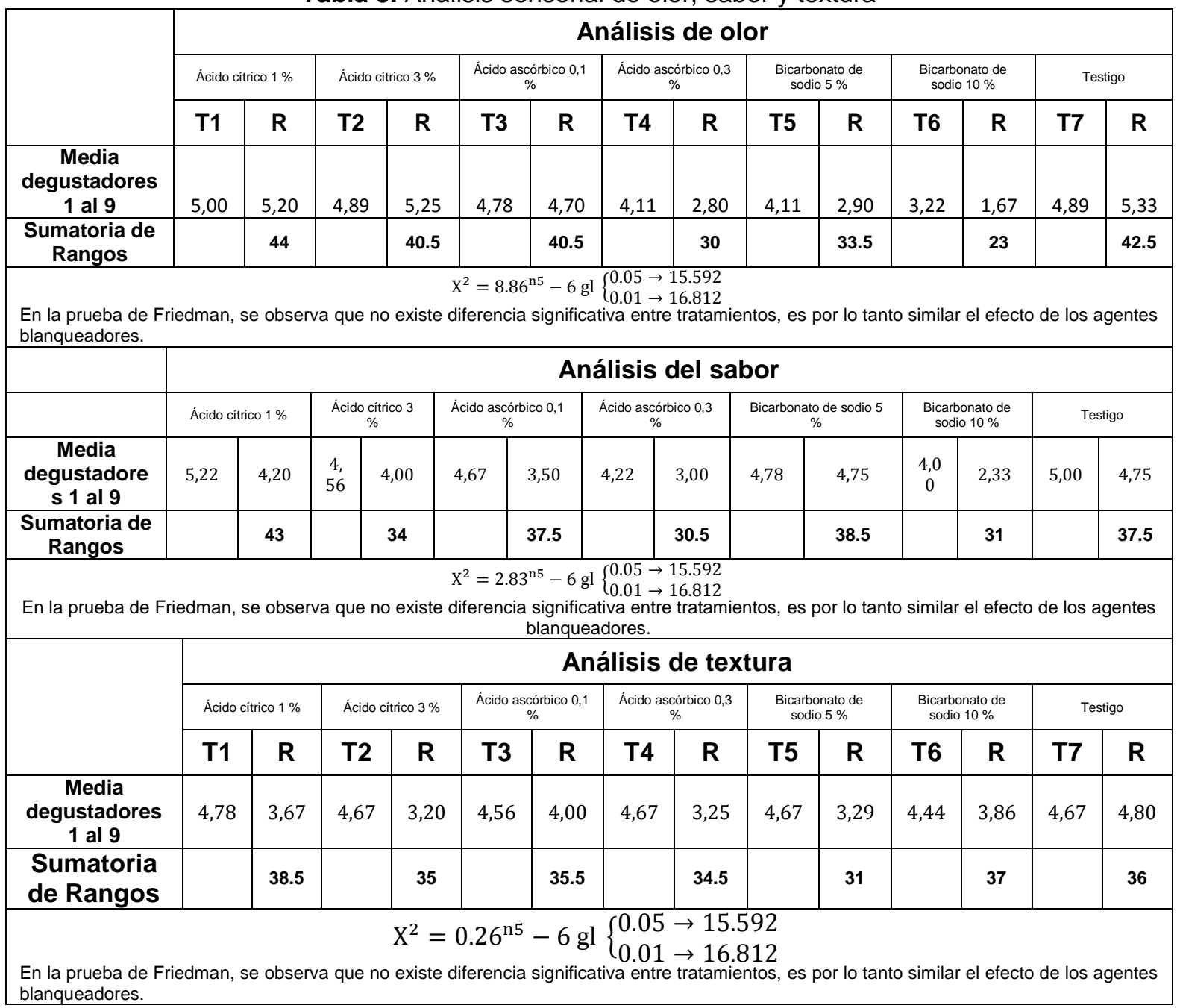

\section{Discusión}

El ácido L-ascórbico, sus isómeros y derivados, son los inhibidores más eficaces y seguros del pardeamiento enzimático (Man, 2002). Este ácido actúa como agente reductor y mantiene los polifenoles en un estado reducido lo cual evita el oscurecimiento en las frutas (Canet \& Álvarez, 2000). El ácido cítrico refuerza determinados sabores, regula el $\mathrm{pH}$ y actúa sinérgicamente con los antioxidantes, inactiva enzimas y previene el pardeamiento, el deterioro del sabor, y actúa como blanqueador (Hughes, 1987). El bicarbonato de sodio, en el caso de las nueces de macadamia es utilizado como un agente blanqueador, no tóxico.

Después de aplicar las soluciones blanqueadoras, como se observó en la Tabla 1, la nuez de macadamia se secó por un tiempo de 4 horas a $60 \stackrel{\circ}{ } \mathrm{C}$.

El tratamiento de ácido ascórbico al $0.3 \%$ que se utilizó en la nuez de macadamia presentó valores superiores de $L^{*}$; existió una diferencia significativa con el bicarbonato de sodio al $5 \%$, este valor indica que la nuez de macadamia presenta una coloración que se acerca al blanco. En cuanto al tratamiento con bicarbonato de sodio al $5 \%$, el valor $\mathrm{L}^{*}$ es el más bajo, en este caso se puede decir que la disminución en la luminosidad de la nuez de macadamia fue porque se dejó en inmersión menos tiempo en la solución con el agente blanqueador. En el estudio realizado por Luna \& Guerrero (2012), los mayores valores de $L^{*}$ corresponden a la nuez de macadamia y nuez de castilla, lo cual indica que 
estos dos fueron los más pálidos, para la nuez de macadamia se obtuvo el valor de 47.22 y para la nuez de castilla se obtuvo el valor de 31.67 .

Cuando existen más de dos tratamientos, se puede utilizar la prueba de los rangos o también llamada de clasificación; en este caso se presentan varias muestras y se les solicita a los jueces que lo clasifiquen u ordenen por orden de preferencia y se suman los rangos para cada muestra. Estas sumas pueden ser interpretadas con la prueba de Friedman (Catania \& Avagnina, 2007). Esta prueba indica que no existe diferencia significativa entre tratamientos lo que permite decir que no hay influencia de los agentes en el sabor de la macadamia.

En la evaluación realizada por Luna y Guerrero (2012), los resultados de la macadamia concuerdan con los reportados por la Sociedad Australiana de macadamia, cuyo valor para la humedad es de $1.4 \%$, estos evaluadores aseguran que el porcentaje de humedad es uno de los factores de mayor importancia que se debe tomar en cuenta ya que puede alterar las propiedades sensoriales de la nuez de macadamia y generar olores y sabores indeseables. Como se observa en la Tabla 3, la nuez de macadamia tiene en el tratamiento de ácido ascórbico al $0.3 \%$, con el proceso de secado al horno durante 4 horas a $60 \stackrel{\circ}{ }$, la humedad más baja de $2.63 \%$ a diferencia del bicarbonato de sodio y ácido cítrico; estos valores se encuentran dentro del rango permitido de humedad.

\section{Conclusiones y recomendaciones}

Luego de realizados los estudios, se determinó que el método óptimo para el blanqueamiento de la nuez de macadamia fue el tratamiento 4, de ácido ascórbico al $0.3 \%$ por tres minutos, ya que este presentó el mayor valor de $L^{*}$, correspondiente al color más blanco. El ácido ascórbico presenta una mayor capacidad captadora de radicales y bloquea la etapa de propagación de la autooxidación. Con el proceso de secado en el horno durante 4 horas a $60^{\circ} \mathrm{C}$, se logró obtener el menor porcentaje de humedad para el Tratamiento 4, el cual se encuentra dentro de los rangos permitidos para humedad en la nuez de macadamia. Al aplicar la prueba de Friedman se pudo demostrar que no hay diferencia significativa entre los tratamientos de ácido cítrico, bicarbonato de sodio y ácido ascórbico en sus respectivas concentraciones, en el análisis sensorial en cuanto a sabor, olor y textura, es decir, que no existe influencia de los agentes de blanqueamiento en las propiedades sensoriales de la macadamia.

\section{Bibliografía}

Aluisa, C. (2013). Proyecto de factibilidad para la exportación de nuez de macadamia natural a Miami, Estados Unidos. Facultad de Ciencias Económicas y Negocios. Universidad Tecnológica Equinoccial, Quito, Ecuador. http://repositorio.ute.edu.ec/handle/123456789/16504

Aulestia, X. (2010). Estudio de Factibilidad para la exportación de confites, elaborados a base de nuez de macadamia ecuatoriana hacia el mercado chileno. Pontificia Universidad Católica del Ecuador Sede Ibarra, Ibarra, Ecuador. dspace.pucesi.edu.ec/bitstream/11010/179/1/T72468.pdf.

Anzaldúa Morales, A. (2005). La evaluación sensorial de los alimentos en la teoría y la práctica. Zaragoza: Acribia. S.A.

Barragán, R. (2012). Métodos estadísticos aplicados al diseño de experimentos, $2^{\circ}$ edición. Quito, Ecuador.

Barreiro, M., Sánchez, R., Ochoa, R., Rodríguez, F., Zavaleta, J., Ortega, C., (2010). Macadamia, la nuez más fina del mundo. Abriendo Surcos, 23.

Canet, W., \& Álvarez, M. (2000). Aplicación de frío de los alimentos. Congelación de los alimentos vegetales. Madrid, 206-251. 
Carvajal, J., Aristizábal, I., Oliveros, C., \& Mejía, J. (2011). Colorimetría del Fruto del Café (Coffea arabica L.). Revista Facultad Nacional de Agronomía, Medellín 64 (2), 6229 - 6240.

Catania, C., \& Avagnina, S. (2007). El Análisis Sensorial. EEA Mendoza. INTA, 15.

Comisión del Codex Alimentarius/FAO/OMS. (2016). Armonización de las disposiciones sobre aditivos alimentarios de normas para productos con las disposiciones pertinentes de la NGAA. Programa Conjunto FAO/OMS sobre normas alimentarias; Comité del Codex sobre aditivos alimentarios. Tema $4 \mathrm{~b}$ del Programa. China.

CODEX STAN 192. (1995). Norma General del Codex para los Aditivos Alimentarios. http://www.codexalimentarius.net/gsfaonline/docs/CXS_192s.pdf

George, D.P., Rigge, R.J., Williams, D.L., \& Hansberry, R.D. (1999). Method for bleaching nuts. Patent US $5871800 \mathrm{~A}$

Erazo, E. (2006). Proyecto de prefactibilidad para la exportación de nuez de macadamia al mercado de Holanda período 2005-2014. Facultad de Ciencias Económicas y Negocios. Universidad Tecnológica Equinoccial, Quito, Ecuador. http://repositorio.ute.edu.ec/browse?type=author\&value=ERAZO+COLMACHI\%2 $\mathrm{C}+\mathrm{E}$ REN+DARIO

FAO. (2006). Fichas Técnicas: Productos frescos y procesados. http://www.fao.org/inpho_archive/content/documents/vlibrary/ae620s/Pfrescos/M ACADAMIA.HTM

Fernández, S. (2012). Toxicología de los Aditivos Alimentarios: Toxicología Alimentaria. Madrid: Díaz de Santos, 454.

Fórmula Estructural Ácido Cítrico. (23 de febrero del 2007). Wikimedia commons, Public domain. http://upload.wikimedia.org/wikipedia/commons/c/c5/Zitronens\%C3\%A4ure_Citric_acid.svg

George, D., Rigge, R., \& Williams, D. (1999). Patente $n^{\circ}$ 5,871,800. United States.

Gil, Á., \& Ruiz, D. (2010). Tratado de Nutrición. E, Composición y Calidad Nutritiva de los Alimentos, Madrid: Panamericana, 201-202.

Hernández, E. (2005). Evaluación Sensorial, 18-20. Bogotá DC.

Hughes, C. (1987). The Additives Guide. Great Britain: Wiley.

Instituto Ecuatoriano de Normalización. (1995). NTE INEN 1 233:95, Granos y cereales. Muestreo.

Lavín, A., Lemus, G., Contreras, M., Negrón, C., \& Orellana, A. (2001). Evaluación de nuevas alternativas de frutales de nuez y mejoramiento de la productividad en Nogal y calidad de la nueces en predios de pequeños productores de la $\mathrm{V}$ región. Curso Frutales de nuez no tradicionales: Macadamia, Pistacho, Pecano, Avellano Europeo , 5.

Luna, J., \& Guerrero, J. (2012). Evaluación de algunos índices Físico y Químicos de aceites extraídos de nuez pecanera, nuez de castilla y macadamia. Ciencia y Tecnología de Alimentos, 35-36.

Man, D. (2002). Caducidad de los alimentos. Zaragoza: Acribia S.A.

Reyes, R., \& Moreno, A. (2009). Proyecto para la industrialización de la macadamia y su influencia en el desarrollo de frutas no tradicionales en el Ecuador. Faculdad de Ciencias Humanísticas y Económicas. Escuela Superior Politécnica del Litoral, Guayaqui, Ecuador. Recuperado el 20 de abril del 2013 de, http://www.dspace.espol.edu.ec/handle/123456789/6826.

Rodríguez Millán, P. S., Silva Ramírez, A. S. \& Carrillo Inungaray, M. L. (2011). Caracterización fisicoquímica del aceite de nuez de macadamia (Macadamia integrifolia). CyTA - Journal of Food, 9:1, 58-64. 